\title{
Does N-Nitrosomelatonin Compete with S-Nitrosothiols as a Long Distance Nitric Oxide Carrier in Plants?
}

\author{
Neha Singh, Harmeet Kaur, Sunita Yadav and Satich C Bhatla* \\ Laboratory of Plant Physiology and Biochemistry, Department of Botany, University of Delhi, India
}

Plants transmit a variety of signaling molecules from roots to aerial parts, and vice-versa, in response to their growth conditions (environment, nutrients, stress factors etc.). A signaling molecule should be produced quickly, induce a defined effect within the cell and be removed or metabolized rapidly when not required. Nitric oxide (NO) plays significant signaling roles in plant cells since it has all the above-stated features. It is a gaseous free radical, can gain or lose an electron, has short half-life $(-30 \mathrm{sec})$ and it can exist in three interchangeable forms, namely the radical $\left(\mathrm{NO}^{*}\right)$, the nitrosonium cation $\left(\mathrm{NO}^{+}\right)$and as nitroxyl radical (NO). $\mathrm{NO}$ is soluble in aqueous as well as lipid phases. It rapidly reacts with oxygen to form $\mathrm{NO} 2$ and can react with other potential signaling molecules as well (eg. superoxide anions $\left(\mathrm{O} 2^{--}\right)$. In the recent past, $\mathrm{NO}$ has been observed to regulate various growth and developmental processes $[1,2]$.

In sunflower seedlings, enhanced NO accumulation in response to salt stress sensed by seedling roots, leads to enhanced tyrosine nitration of proteins in seedling cotyledons [2]. Although investigations have been undertaken in animal systems to examine targeted delivery of NO [3], mechanisms of transcellular migration of $\mathrm{NO}$ from one tissue system in the plant body (eg. roots) to another long distance region (e.g. shoots) are so far not fully understood and require deeper investigations. In order to act as a long distance signaling molecule, NO interacts with certain carrier molecules. In this context, endogenous $S$-nitrosothiols have been suggested to be responsible for NO transport [4].

\section{$S$-Nitrosothiols-versatile molecules as NO carriers}

$S$-nitrosothiols (SNOs), which are formed by the reaction of NO with protein or non-protein sulphydryl-containing compounds, facilitate various important physiological functions performed by NO $[5,6]$. SNOs are generated in vivo under physiological conditions to reach a concentration of up to $1 \mu \mathrm{M}$ [4]. The in vivo half-life of NO is very short as compared to that of SNOs which are generally more stable in solution [7-10]. SNOs have the ability to transport, store and deliver NO. They can also participate in post-translational modifications triggered by cell signaling and stress conditions [11]. SNOs are involved in many important biological reactions which includes $\mathrm{NO}$ release, transnitrosation, $S$-thiolation, in addition to direct actions $[5,12]$. $S$-nitrosylation of cysteine thiols of proteins is a significant redox-based post-translational modification $[6,12]$. SNOs are divided into two families: low-molecular-mass $S$-nitrosothiols (LMM-SNOs) and highmolecular mass $S$-nitrosothiols (HMM-SNOs). S-nitrosoglutathione (GSNO), $S$-nitrosocysteine (CySNO) and $S$-nitrosocysteinylglycine (GlyCySNO) are LMM-SNOs, of which GSNO is the most abundant in biological systems [13].

It is generated by the interaction of $\mathrm{NO}$ and reduced glutathione (thiol tripeptide, $\gamma$-glutamyl cysteinyl glycine; GSH) by a process called $S$-nitrosylation. The reaction takes place either via $\mathrm{N}_{2} \mathrm{O}_{3}$ formation or by the addition of $\mathrm{NO}$ to a glutathionyl radical formed during this reaction [14]. GSNO has significant physiological relevance since it is thought to function as an intracellular mobile reservoir of NO bioactivity both in animals and plant cells [12,15-18]. HMM-SNOs are produced by NO binding to sulfhydryl (-SH) groups present in specific cysteine residues of proteins [13].

Determination of GSNO in plant samples still presents a challenge due to several technical obstacles and cumbersome sample preparation procedures. It can also be affected by light, metal-catalyzed SNO decomposition, enzymatic degradation catalyzed by GSNO reductase, and a reduction in the $S$-NO bond caused by reductants and endogenous thiols [13]. Two different approaches to detect GSNO have been reported in higher plants: immunohistochemical analysis using commercial antibodies against GSNO and liquid chromatographyelectrospray/mass spectrometry (LC- ES/MS) [19-21].

\section{$\mathrm{N}$-Nitrosomelatonin- probable competitor of $S$-Nitro- sothiols for long distance transport of nitric oxide in plants}

Recent investigations in the author's laboratory have demonstrated the critical role of melatonin (a neurotransmitter in animals) in transducing abiotic stress-induced signals from seedling roots to the aerial parts [22]. This is achieved by enhanced melatonin accumulation in seedling roots and cotyledons, which interferes with auxin action on growth modulation. Melatonin modulates auxin action in cell elongation through its biosynthesis from tryptophan which is also the precursor for the biosynthesis of indole-3-acetic acid. Melatonin is also capable of scavenging reactive oxygen species (ROS) accumulated in the cells in response to stress [23]. It has been reported to increase the activities of various ROS scavenging enzymes [24]. Melatonin molecule consists of an electron-rich indole moiety and two side chain groups, namely 5-methoxy group $\left(\mathrm{H}_{3} \mathrm{CO}\right)$ and 3 -amide group $\left(\mathrm{CH}_{3} \mathrm{COONH}\right)$. Its high resonance stability, electroreactivity and low activation energy barriers make melatonin a potent free radical scavenger [25,26]. Its side chains also have a significant contribution in the antioxidative properties of the melatonin molecule. Carbonyl moiety present in the functional group $(\mathrm{N}-\mathrm{C}=\mathrm{O})$ of $\mathrm{C} 3$ amide side chain is crucial for its ability to quench ROS. Melatonin is an efficient ROS scavenger since up to four or more molecules of ROS can be scavenged by a single melatonin molecule [27]. The mechanisms of ROS scavenging by melatonin involves processes, such as electron donation to form melatoninyl cation radical, hydrogen donation from nitrogen atom, nitrosation, addition, substitution and nitrosation reactions [25, 28-29]. Melatonin may also directly scavenge $\mathrm{H}_{2} \mathrm{O}_{2}$ and help in maintaining a steady state concentration of $\mathrm{H}_{2} \mathrm{O}_{2}$ [30]. Melatonin biosynthesis and its regulation involves the activity

*Corresponding author: Satich C Bhatla, Laboratory of Plant Physiology and Biochemistry, Department of Botany, University of Delhi, India, Tel: 9818737090 ; E-mail: bhatlasc@gmail.com

Received: January 20, 2016; Accepted: March 28, 2016; Published March 31, 2016

Citation: Singh N, Kaur H, Yadav S, Bhatla SC (2016) Does N-Nitrosomelatonin Compete with S-Nitrosothiols as a Long Distance Nitric Oxide Carrier in Plants? Biochem Anal Biochem 5: 262. doi:10.4172/2161-1009.1000262

Copyright: ( 2016 Singh N, et al. This is an open-access article distributed under the terms of the Creative Commons Attribution License, which permits unrestricted use, distribution, and reproduction in any medium, provided the original author and source are credited. 
of enzymes-Serotonin $N$-acetyltransferase (SNAT, E.C. 2.3.1.87) and hydroxyindole-O-methyltransferase (HIOMT, E.C.2.1.1.4). HIOMT is a rate limiting enzyme and regulates the final step of melatonin biosynthesis from $\mathrm{N}$-acetyl serotonin $[31,32]$. Recent findings from the author's laboratory have shown a $72 \%$ increase in HIOMT activity in response to $120 \mathrm{mM} \mathrm{NaCl}$ stress in 2 day old, dark-grown sunflower seedling cotyledons [22]. Transcriptomic analysis in Arabidopsis has shown that 183 genes are associated with melatonin-induced changes in hormonal signaling [33]. Among the major plant hormones, indole3-acetic acid (IAA) is structurally similar to melatonin [34]. Auxin responsive genes are both up- and down-regulated in response to melatonin. Auxin transport and homoeostasis thus seem to be under the precise regulation by melatonin [33].

Melatonin is an $\mathrm{N}$-terminal blocked tryptophan derivative which can be easily nitrosated via various mechanisms at the nitrogen atom of the indole ring to give $N$-nitrosomelatonin (NOMela). Melatonin can be nitrosated at physiological $\mathrm{pH}$ by employing four nitrosating entities namely, $\mathrm{N}_{2} \mathrm{O}_{3}$, nitroxyl ions, peroxynitrite, or $\mathrm{N}$-nitroso- $\mathrm{N}$ acetyl tryptophan [35] (Figure 1). Nitrosation of melatonin by NO occurs only under aerobic conditions since $\mathrm{N}$-terminal blocked tryptophan derivatives do not react with $\mathrm{NO}$ in the absence of oxygen. Peroxynitrite-induced nitrosation of melatonin proceeds via $\mathrm{N}_{2} \mathrm{O}_{4}$ formation and it nitrates melatonin at various positions $(\mathrm{N}-1, \mathrm{C}-4$, C-6). Nitrosation of melatonin by peroxynitrite has, however, been observed to be a low efficiency process. Nitrosation of melatonin is also possible through its interaction with nitroxyl moiety. This process is, however, not yet fully understood. None of the above-stated nitrosation routes of melatonin have been reported to be effective in in vivo because $\mathrm{N}$-terminal blocked tryptophan derivatives present in proteins are in the orders of magnitude higher concentrated than melatonin and are only slightly effective than melatonin in scavenging reactive nitrogen species (RNS) and ROS. Generation of NOMela through the transfer of $\mathrm{NO}^{+}$function via a biomolecular reaction from a $\mathrm{N}$-nitroso $\mathrm{N}$-terminal blocked tryptophan derivative to melatonin at physiological $\mathrm{pH}$ has,

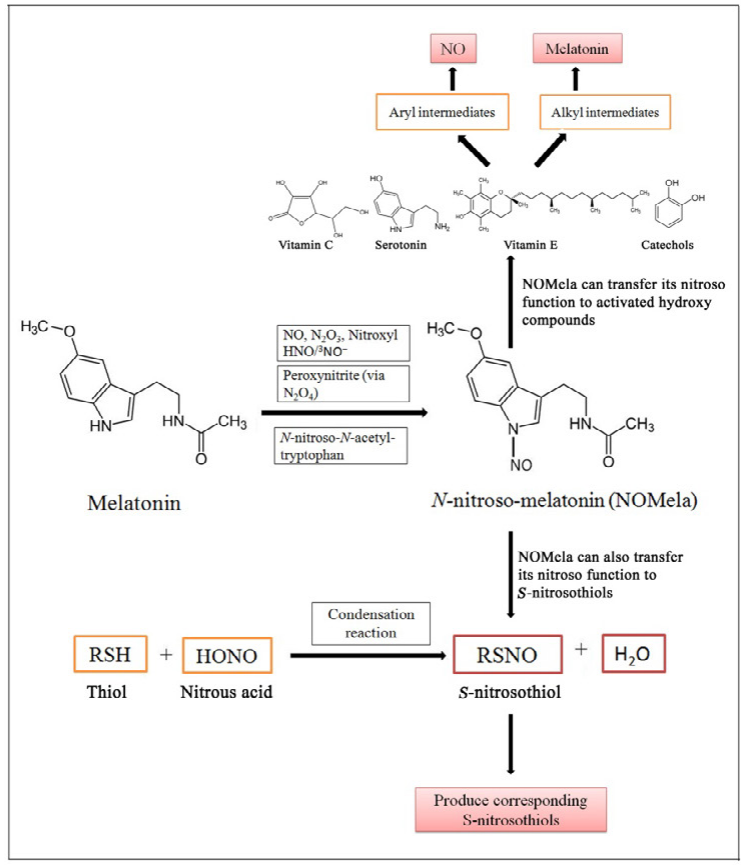

Figure 1: Possible routes of $\mathrm{N}$-Nitrosomelatonin (NOMela) formation and NO release.

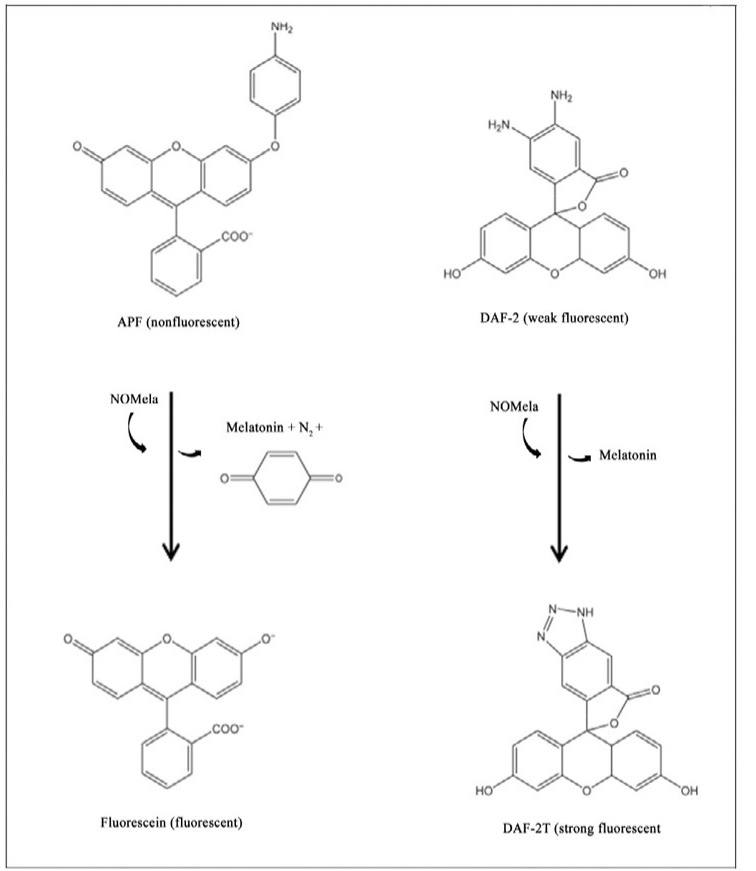

Figure 2: Mechanisms of detection of NOMela using fluorescence techniques.

however, been reported. This reaction is analogous to transnitrosation reaction between $S$-nitrosothiols and thiols, and is a reversible reaction.

\section{SNOs or NOMela- biochemical features and limitations as NO carriers}

NOMela can be quantitatively determined both by direct and indirect methods [35]. In a pure sample, NOMela has a $\lambda$ max absorbance at 346 $\mathrm{nm}$. This feature can be used to determine NOMela concentrations in the range of $5-100 \mu \mathrm{M}$. However, if the samples contain other compounds with same $\lambda \max (346 \mathrm{~nm})$, purification of NOMela by capillary zone electrophoresis or HPLC is suggested. The indirect methods for NOMela detection rely on the production of certain fluorescent derivatives (Figure 2). Thus, NOMela can react with aromatic amines, such as amino phenyl fluorescein (APF), and 4,5-diaminofluorescein (DAF-2) to yield fluorescent dyes fluorescein and triazolfluorescein (DAF-2D), respectively. These aromatic amines can thus be used as reporter molecules for NOMela detection. The major limitation of this procedure for NOMela detection is that the fluorescence of fluorescein and DAF-2D is significantly quenched by proteins. Another reporter molecule, $N$-methyl-4-hydrazino-7-nitrobenzofurazan (MNBDH), however, reacts with NOMela and the fluorescence of the reaction product $N$-methyl-4-amino-7-nitrosobenozfurazan (MNBDA) is not quenched by proteins. Furthermore, $S$-nitrosothiols do not react with MNBDH in the presence of EDTA. Thus, it is possible to detect NOMela using MNBDH even in the presence of $S$-nitrosothiols, since EDTA complexes with catalytically active transition metal ions required for decomposition of $S$-nitroso compounds. Additionally, working at $\mathrm{pH} 11$ (where the rate of hydrolysis reactions is about eight fold lower than at $\mathrm{pH}$ 7.4) can reduce the influence of other competing hydrolysis reactions.

$\mathrm{N}$-nitrosomelatonin (NOMela) can act as a NO releasing compound as it can efficiently transfer its nitroso function $\left(\mathrm{NO}^{+}\right)$to activated hydroxy compounds (eg. vitamin C, vitamin E, catechols or serotonin) with the generation of melatonin and $\mathrm{NO}$ via alkyl and aryl nitrite 
intermediates, respectively [36-40]. NOMela can also shift its nitroso function to $S$-nitrosothiols to yield corresponding $S$-nitosothiols. It is likely to instantaneously release melatonin, $\mathrm{NO}$, and $S$-nitrosothiols, thereby avoiding the yield of nitoxyl radicals when $S$-nitrosothiols react with thiols [41]. NOMela is highly sensitive and unstable in light. In aqueous solution, N-NO bond of NOMela is broken in a hemolytic manner to produce NO and aminyl radical [42]. NO-released from NOMela is independent of the composition of the buffer system. In the case of $S$-nitrosothiols like GSNO, NO-generation is, however, dependent on buffer composition [4]. LMM-SNOs such as GSNO react with thiols reversibly but not regiospecifically, thus leading to the production of toxic nitroxyl compound in a prominent side reaction. In contrast, NOMela reacts with thiols in an irreversible, regiospecific manner and, is therefore, able to more efficiently transnitrosate cysteinyl residues in proteins. In fact, in a pure chemical system, NOMela has been found to be 10 -fold more effective than $S$-nitrocysteine in nitrosating the catalytically active cysteinyl residue of glyceraldehyde-3-phosphate dehydrogenase (GAPDH) [43-45]. A major problem with the use of almost every $\mathrm{NO}$ donor under aerobic condition is that they produce noxious oxidizing nitrogen oxygen species upon NO release. However, the production of these harmful species is automatically limited by the use of NOMela as NO-releasing compound. Since NOMela releases melatonin together with the generation of $\mathrm{NO}$, therefore it works as a more efficient antioxidant [4].

\section{Probable long distance routes of transport of SNOs and NOMela}

Both xylem and phloem elements have certain unique features some of which are responsible for determining the nature of biomolecular transport across them from one region to another region in the plant. Mature vessels and tracheids are dead, hollow, lack plasma membrane and have a rigid cell wall impregnated with lignin. Absence of lipid membranes and hydrogel nature of pectin membranes of pits can regulate transport of metabolites through xylem elements. The absence of lipid membranes in vessels and tracheids enhances hydraulic conductance several folds as compare to cells intact with plasma membrane. Pit membrane consists of cellular microfibrils and a pectin/hemicellulose matrix with a number of pores $(5-20 \mathrm{~nm}$ diameter), whereas the pectin component of pit membrane can respond like a hydrogel and lignification provides water proofing of cell walls. During development, sieve elements lose their nuclei and tonoplast. Mature sieve elements generally lack microfilaments, microtubules, golgi bodies and ribosomes but they contain mitochondria, plastids, smooth endoplasmic reticulum and their walls become non-lignified. Additionally, the parietal cytoplasm within the sieve tube elements contains phloem filament protein (PP1) and phloem lectin (PP2).

Based on the above-stated biochemical features of SNOs and NOMela, future investigations are likely to decipher the anatomical routes of SNOs and NOMela migration from one tissue/organ to another.

\section{Perspectives}

Based on the in depth analysis of the unique chemical properties of $\mathrm{N}$-nitrosomelatonin and $\mathrm{S}$-nitrosothiols and their physiological and biochemical relevance in serving as potential competitors for long distance transport of NO in plants, the major challenges likely to be faced by plant biologists reside in precise applications of the available and upcoming technologies for a better understanding the significance of the two biomolecules in plant cells. Applications of fluorescence technologies, such as confocal imaging of live cells, are more likely to yield faster, accurate and precisely interpretable results on the spatiotemporal distribution and quantification of the two NO-transporting molecules in plant cells. Methodologies for such imaging have, however, to be developed with due caution keeping in view the $\mathrm{pH}$ sensitivity and stability of $S$-nitrosothiols and NOMela under the conditions of experimental analysis. Several instrument-dependent factors are also likely to limit the sought after information from live cell imaging. These include the ability to detect biomolecules above the background and instrument noise and the spatial resolution of the imaging system, Multiphoton imaging, which has revolutionized in in vivo imaging in animals, is strongly recommended for currently suggested applications in plant cells to reduce photobleaching of fluorescent biomolecules and generate images through highly focused excitation and emission. Surface plasma resonance (SPR) has revolutionized life science research by enabling real time detection and monitoring of biomolecular binding events. When used in an imaging mode, SPR can be very useful for quantifying and visualizing both fixed and live cells at low levels of incident light and be able to quantify very small amounts (up to $3 \mathrm{ng}$ $\mathrm{cm}^{-2}$ ) of $S$-nitrosothiols and NOMela from plant tissue. Again, as stated earlier, all this requires a very precise optimization of the protocol. This novel field of investigation marks an interface of chemistry and biology, and will benefit a lot from collaboration among the current generations of chemists, biochemists and plant physiologists. If, through the dedicated and persistent efforts, we can exploit the above-stated high end imaging technologies, the findings obtained there from are likely to revolutionize our current understanding of the NO-mediated long distance signaling mechanisms in plants, more so in plants exposed to a variety of biotic and abiotic stress conditions. Such an enhancement of our understanding of the mechanisms of stress tolerance in plants shall benefit plant biologists in particular and mankind in general, in their further pursuit to unravel novel survival strategies adopted by plants under harsh environmental conditions.

\section{References}

1. David A, Yadav S, Bhatla SC (2010) Sodium chloride stress induces nitric oxide accumulation in root tips and oil body surface accompanying slower oleosin degradation in sunflower seedlings. Physiol Plant 140: 342-354.

2. David A, Yadav S, Baluška F, Bhatla SC (2015) Nitric oxide accumulation and protein tyrosine nitration as a rapid and long distance signalling response to salt stress in sunflower seedlings. Nitric Oxide 50: 28-37.

3. Gross SS (2001) Targeted delivery of nitric oxide. Nature 409: 577-578.

4. Berchner-Pfannschmidt U, Tug S, Trinidad B, Becker M, Oehme F, et al (2008) The impact of $\mathrm{N}$-nitrosomelatonin as nitric oxide donor in cell culture experiments. J Pineal Res 45: 489-496.

5. Hogg N (2000) Biological chemistry and clinical potential of S-nitrosothiols Free Radical Bio Med 28: 1478-1486

6. Foster MW, McMahon TJ, Stamler JS (2003) S-nitrosylation in health and disease. Trends Mol Med 9: 160-168.

7. Feechan A, Kwon E, Yun BW, Wang Y, Pallas JA, et al. (2005) A central role for S-nitrosothiols in plant disease resistance. Proc Natl Acad Sci USA 102 8054-8059.

8. Gaston B, Singel D, Doctor A, Stamler JS (2006) S-nitrosothiol signaling in respiratory biology. Am J Respir Crit Care Med 173: 1186-1193.

9. Corpas FJ, Chaki M, Fernandez-Ocana A, Valderrama R, Palma JM, et al (2008) Metabolism of reactive nitrogen species in pea plants under abiotic stress conditions. Plant Cell Physiol 49: 1711-1722.

10. Lindermayr C, Sell S, Durner J (2008) Generation and detection of S-nitrosothiols. Methods Mol Biol 476: 217-229.

11. Lindermayr C, Durner J (2009) S-Nitrosylation in plants: pattern and function J Proteomics 73: 1-9.

12. Stamler JS, Lamas S, Fang FC (2001) Nitrosylation: the prototypic redox based signaling mechanism. Cell 106: 675-683. 
Citation: Singh N, Kaur H, Yadav S, Bhatla SC (2016) Does N-Nitrosomelatonin Compete with S-Nitrosothiols as a Long Distance Nitric Oxide Carrier in Plants? Biochem Anal Biochem 5: 262. doi:10.4172/2161-1009.1000262

13. Corpas FJ, Alché JD, Barroso JB (2013) Current overview of S-nitrosoglutathione (GSNO) in higher plants. Front Plant Sci 4: 126.

14. Broniowska KA, Diers AR, Hogg N (2013) S-Nitrosoglutathione. Biochim Biophys.Acta 1830: 3173-3181.

15. Durner J, Gow AJ, Stamler JS, Glazebrook J (1999) Ancient origins of nitric oxide signaling in biological systems. Proc Natl Acad Sci USA 96: 1420614207.

16. Diaz M, Achkor H, Titarenko E, Martinez MC (2003) The gene encoding glutathione-dependent formaldehyde dehydrogenase/GSNO reductase is responsive to wounding, jasmonic acid and salicylic acid. FEBS Lett 543: 136-139.

17. Ng ES, Kubes $P$ (2003) The physiology of $S$-nitrosothiols: carrier molecules for nitric oxide. Can J Physiol Pharmacol 81: 759-764.

18. Wang Y, Yun BW, Kwon E, Hong JK, Yoon J, et al. (2006) S-Nitrosylation: an emerging redox-based post-translational modification in plants. J Exp Bot 57: 1777- 1784 .

19. Barroso JB, Corpas FJ, Carreras A, Rodríguez- Serrano M, Esteban FJ, et al. (2006) Localization of S-nitrosoglutathione and expression of S-nitrosoglutathione reductase in pea plants under cadmium stress. J Exp Bot 57: 1785-1793.

20. Valderrama R, Corpas FJ, Carreras A, Fernández- Ocaña A, Chaki M, et al. (2007). Nitrosative stress in plants. FEBS Lett 581: 453-461.

21. Airaki M, Sánchez-Moreno L, Leterrier M, Barroso JB, Palma JM, et al. (2011) Detection and quantification of S-nitrosoglutathione (GSNO) in pepper (Capsicum annuum L.) plant organs by LC-ES/MS. Plant Cell Physiol 52 2006-2015.

22. Mukherjee S, David A, Yadav S, Baluška F, Bhatla SC (2014) Salt stressinduced seedling growth inhibition coincides with differential distribution of serotonin and melatonin in sunflower seedling roots and cotyledons. Physiol Plant 152: 714-728.

23. Kaur H, Mukherhjee S, Baluska F, Bhatla SC (2015) Regulatory roles of serotonin and melatonin in abiotic stress tolerance in plants. Plant Signal Behav 10: 1049788.

24. Reiter RJ, Tan DX, Acuna-Castroviejo D, Burkhardt S, Karbownik M (2000) Melatonin mechanisms and actions as an antioxidant. Curr Top Biophys 24:171-83

25. Tan DX, Chen LD, Poeggeler B, Manchester LC, Reiter RJ (1993) Melatonin: a potent, endogenous hydroxyl radical scavenger. Endocrine J 1:57-60.

26. Tan DX, Reiter RJ, Manchester LC, Yan M, El-Sawi M, et al. (2002) Chemical and physical properties and potential mechanisms: melatonin as a broad spectrum antioxidant and free radical scavenger. Curr Top Med Chem 2: 181 197.

27. Afreen F, Zobayed SMA, Kozai T (2006) Melatonin in Glycyrrhiza uralensis: response of plant roots to spectral quality of light and UV-B radiation. J Pineal Res 41: 108-115.

28. Tan DX, Manchester LC, Reiter RJ, Plummer BF, Hardies LJ, et al. (1998) A novel melatonin metabolite, cyclic 3-hydroxymelatonin: a biomarker of in vivo hydroxyl radical generation. Biochem Biophys Res Commun 253: 614-620.

29. Reiter RJ, Tan DX, Terron MP, Flores LJ, Czarnocki Z (2007) Melatonin and its metabolites: new findings regarding their production and their radical scavenging actions. Acta Biochim Pol 54: 1-9.

30. Tan DX, Manchester LC, Reiter RJ, Qi WB, Karbownik M, et al. (2000) Significance of melatonin in antioxidative defence system: reactions and products. Biol Signals Recept 9:137-159.

31. Park S, Byeon Y, Back K (2013) Functional analyses of three ASMT gene family members in rice plants. J Pineal Res 55: 409-415.

32. Byeon Y, Back K (2014) Melatonin synthesis in rice seedlings in vivo is enhanced at high temperatures and under dark conditions due to increased serotonin acetyltransferase and $\mathrm{N}$-acetylserotonin methyltransferase activities. J Pineal Res 56: 189-195.

33. Weeda S, Zhang N, Zhao XL, Ndip G, Guo YD, et al. (2014) Arabidopsis transcriptome analysis reveals key roles of melatonin in plant defense systems. PLoS One 9: 93462.

34. Baluska F, Mancuso S (2013) Root apex transition zone as oscillatory zone. Front Plant Sci 4:354.

35. Kirsch M, de Groot H (2009) N-Nitrosomelatonin: synthesis, chemical properties, potential prodrug. J Pineal Res 46: 121-127.

36. Kirsch M, Fuchs A, De Groot $\mathrm{H}$ (2003) Regiospecific nitrosation of N-(terminal) blocked tryptophan derivatives by $\mathrm{N} 2 \mathrm{O} 3$ at physiological $\mathrm{pH}$. J Biol Chem 278 : 11931-11936

37. Kytzia A, Korth HG, Sustmann R, de Groot H, Kirsch M (2006) On the mechanism of the ascorbic acid-induced release of nitric oxide from nitrosated tryptophan derivatives. Scavenging of NO by ascorbyl radicals. Chem Eur J 12: 8786-8797.

38. Muller C, Korth HG, De Groot H, Kirsch M (2007) Reaction of Vitamin E compounds with $\mathrm{N}$-nitrosated tryptophan derivatives and its analytical use. Chem Eur J 13: 7532- 7542 .

39. Kytzia A, Korth HG, De Groot H, Kirsch M (2006) Catecholamine induced release of nitric oxide from $\mathrm{N}$-nitrosotryptophan derivatives: a non-enzymatic way of catecholamine-oxidation. Org Biomol Chem 4: 257-267.

40. Kopczak A, Korth HG, De Groot H, Kirsch M (2007) N-nitrosomelatonin releases nitric oxide in the presence of serotonin and its derivatives. J Pineal Res 43: 343-350.

41. Sonnenschein K, De Groot H, Kirsch M (2004) Formation of S-nitrosothiols from regiospecific reaction of thiols with $\mathrm{N}$-nitrosotryptophan-derivatives. J Bio Chem 279: 45433-45440.

42. De Biase PM, Turjanski AG, Estrin DA, Doctorovich F (2005) Mechanisms of $\mathrm{NO}$ release by $\mathrm{N}$-nitrosomelatonin: nucleophilic attack versus reducing pathways. J Org Chem 70: 5790-5798.

43. Wong PSY, Hyun J, Fukuto JM, Shirota FN, de Master EG (1998) Reaction between S-nitrosothiols and thiols: generation of nitroxyl (HNO) and subsequent chemistry. Biochem 37: 5362-5371.

44. Liebeskind S, Korth HG, De Groot H, Kirsch M (2008) Release of nitric oxide ammonium and nitroxyl by nitroso-dithiols is dependent on the extent of nitrosation. Evidence that dinitroso-dithiothreitol acts only as an nitric oxide releasing compound. Org Biomol Chem 6:2560-2573.

45. Kirsch M, De Groot H (2008) N-Nitrosomelatonin outcompensates S-Nitrosocysteine in inhibiting glyceraldehyde 3-Phosphate dehydrogenase: first evidence that $\mathrm{N}-$ Nitrosomelatonin can modify protein function. J Pineal Res 44: 244-424. 